Artículo

\title{
Resistencia antimicrobiana de Salmonella spp aisladas de canales de cerdo obtenidas de dos tipos de rastros en Jalisco, México
}

\author{
Vicente Vega-Sánchez ${ }^{\text {a }}$ \\ Jeannette Barba-León ${ }^{\text {* }}$ \\ Delia Guillermina González-Aguilar ${ }^{\mathrm{b}}$ \\ Elisa Cabrera-Díaz ${ }^{\mathrm{b}}$ \\ Carlos Pacheco-Gallardo ${ }^{b} \dagger$ \\ Adriana Guadalupe Orozco-García ${ }^{\text {b }}$
}

${ }^{a}$ Universidad Autónoma del Estado de Hidalgo, Instituto de Ciencias Agropecuarias, Hidalgo, México.

b Universidad de Guadalajara, Centro Universitario de Ciencias Biológicas y Agropecuarias, Departamento de Salud Pública, Camino Ramón Padilla Sánchez No. 2100 Nextipac, Zapopan. 45200, Jalisco México.

* Autor de correspondencia: jeannette.barba@academicos.udg.mx

\section{Resumen:}

Salmonella es una de las principales bacterias que originan enfermedades transmitidas por alimentos. El estudio de la resistencia mostrada por Salmonella a diferentes antimicrobianos ha cobrado importancia en los últimos años debido a las complicaciones en el tratamiento de las infecciones causadas por cepas resistentes. Este estudio muestra el perfil de resistencia de cepas de Salmonella aisladas en dos rastros que se diferencian en los procesos de sacrificio y faenado del ganado porcino. Los resultados de este estudio muestran que el rastro que ha implementado y cumple con los procesos sanitarios de obtención de la carne tiene una menor cantidad de aislamientos de Salmonella (1.3\%), que aquel cuyas prácticas de higiene son menos rigurosas $(46.8 \%)(P<0.05)$. Los principales serotipos de Salmonella encontrados fueron London (44.7\%), Anatum (15.8 $\%$ ), Agona, Muenchen y Typhimurium (7.9\%). La resistencia a los aminoglucósidos (100 $\%)$, tetraciclinas $(73.7 \%)$ y ciprofloxacina $(44.7 \%)$ fueron predominantes en los 
aislamientos evaluados. El $66.6 \%$ de las cepas evaluadas fueron resistentes a 3 ó 4 clases diferentes de antimicrobianos, y se encontró la presencia del gen que codifica para la integrasa 1. Los resultados muestran que Salmonella ha adquirido diferentes elementos genéticos que la vuelven resistente a diferentes clases de antimicrobianos, complicando el tratamiento de una infección causada por este patógeno. Así mismo, sugieren que la implementación y cumplimiento de los procesos sanitarios de obtención de la carne del ganado porcino disminuyen los aislamientos de Salmonella en las canales.

Palabras clave: Rastros, Salmonella, Canales de cerdo, Serotipos, Resistencia antimicrobiana.

Recibido: 20/05/2019

Aceptado: 25/11/2019

\section{Introducción}

El estudio de la frecuencia de bacterias patógenas en alimentos, así como el análisis de la resistencia a antimicrobianos que poseen, es uno de los temas de estudio que han cobrado relevancia en años recientes debido a la dificultad observada en el tratamiento de las enfermedades ocasionadas por bacterias patógenas resistentes ${ }^{(1)}$. En la reunión de expertos convocada por la Organización de las Naciones Unidas para la Alimentación y la Agricultura (FAO, por sus siglas en inglés) y la Organización Mundial de la Salud (OMS) que se llevó a cabo en el 2018, trataron el tema de los patógenos resistentes en alimentos, su papel en el ambiente, los cultivos y los biocidas. Estableciendo cinco áreas prioritarias de vigilancia, entre las que destacan la presencia de bacterias resistentes, residuos tóxicos y genes de resistencia en el ambiente cercano a la producción de alimentos; el uso frecuente de anitimicrobianos en la producción de alimentos y su relación con el desarrollo de bacterias resistentes, y la integración de sistemas de supervisión integrada, que vigilen las prácticas que se siguen en los procesos de todas las etapas de la producción y distribución de alimentos ${ }^{(2)}$. Al respecto, Estados Unidos de Norteamérica y los países que conforman la Unión Europea, han implementado sistemas de supervisión como el Sistema Nacional de Monitoreo de la Resistencia Antimicrobiana (NARMS, por sus siglas en inglés) y la Autoridad Europea en Seguridad Alimentaria (EFSA, por sus siglas en inglés) $^{(3,4)}$.

Existe una gran cantidad de reportes que muestran que la frecuencia de bacterias patógenas y resistentes en alimentos es un problema que se presenta en diversos países. La cantidad de cepas patógenas resistentes reportadas es diferente en cada región del 
mundo, pero dicha cantidad está en relación a la implementación y seguimiento de medidas de control en los procesos de producción de alimentos ${ }^{(4-6)}$.

El consumo de carne de cerdo en México se ha incrementado debido a que su precio es competitivo con el reportado para otro tipo de carnes como las de ave o bovino. Para el

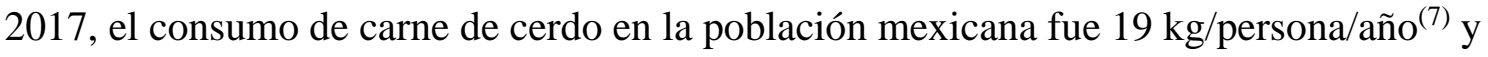
a nivel internacional en 2018 se estimó que México ocuparía el décimo lugar en la producción mundial de carne de cerdo, con una aportación de 1.5 millones de toneladas. Para ese mismo año, se pronosticó que la producción anual de carne de cerdo crecería en un $2.3 \%$ con una producción de 113.5 millones de toneladas, siendo Jalisco, Sonora, Puebla, Yucatán y Veracruz las entidades donde se concentraría el $69.4 \%$ de la producción nacional $^{(8)}$.

En México operan dos tipos de rastros, los rastros Tipo Inspección Federal (TIF) y los rastro no certificados de competencia municipal $(\mathrm{RNC})^{(9)}$. El rastro TIF es una instalación de sacrificio de animales de abasto e industrializadores de productos y subgrupos cárnicos, que es objeto de una inspección sanitaria permanente por parte de la Secretaría de Agricultura y Desarrollo Rural (SADER) donde se garantizan productos de óptima calidad higiénico sanitaria ${ }^{(10)}$. Por su parte los rastros RNC son instalaciones propiedad del municipio, que se destinan al sacrificio y faenados de animales de abasto y que deben de cumplir con lo establecido en la NOM-194-SSA-2004 ${ }^{(11)}$.

\section{Material y métodos}

El objetivo de este estudio fue aislar Salmonella enterica en canales de cerdo procesadas en dos tipos de rastro (TIF y RNC) y analizar la resistencia de los aislamientos contra 10 antimicrobianos de uso humano y veterinario.

Para este estudio se recolectaron 159 muestras de la superficie de canales de cerdo, 79 de ellas de un establecimiento RNC y 80 de un tipo TIF, ubicados en las zonas Centro y Sur de los Altos de Jalisco, respectivamente. El periodo del muestreo comprendió los meses de octubre del 2013 a mayo del 2014 y se procesaron ocho muestras provenientes de cada rastro cada quince días. Las muestras se colectaron de la superficie de canales seleccionadas aleatoriamente en cada visita. Con una esponja estéril humedecida con 10 $\mathrm{ml}$ de agua peptonada amortiguada (APA, DB), se frotó una superficie de $100 \mathrm{~cm}^{2} \mathrm{de}$ cada una de las regiones del vientre (panza), jamón y papada para una superficie total de $300 \mathrm{~cm}^{2}$. Las esponjas se introdujeron en bolsas estériles y se colocaron en hieleras con refrigerantes para su transporte al laboratorio de Inocuidad de Alimentos de la Universidad de Guadalajara, donde se analizaron de acuerdo a lo especificado por la técnica MLG 4.04 ${ }^{(12)}$. 
El aislamiento de Salmonella spp se llevó a cabo de las esponjas recolectadas adicionándoles $50 \mathrm{ml}$ de APA para obtener un volumen total de $60 \mathrm{ml}$, posteriormente la muestra se homogenizó en un homogenizador peristáltico (BagMixer ${ }^{\circledR}$ ) por 1 min y se incubó a $35 \pm 2{ }^{\circ} \mathrm{C}$ durante 20 a $24 \mathrm{~h}$. De cada muestra homogenizada se transfirieron 0.1 $\mathrm{ml}$ en $9.9 \mathrm{ml}$ de caldo tetrationato (CTT) (BD) y $1 \mathrm{ml}$ en $9 \mathrm{ml}$ de caldo Rappaport Vassiliadis modificado (mRV) (BD). Los caldos selectivos se incubaron a $42 \pm 1{ }^{\circ} \mathrm{C}$ durante $24 \mathrm{~h}$. La discriminación de muestras se realizó por medio de la reacción de cadena de la polimerasa (PCR) amplificando los genes invA y fimA usando condiciones previamente descritas ${ }^{(13)}$. Transcurridas las $24 \mathrm{~h}$ de incubación, se tomaron $500 \mu \mathrm{l}$ del CTT y $500 \mu \mathrm{l}$ del mRV para purificar ADN con el kit comercial Wizard (PROMEGA) siguiendo las condiciones del fabricante. Las muestras en donde se observó la amplificación de los genes invA y fimA se continuaron para aislamiento selectivo en agar verde brillante sulfa (VBS, BD) y en Agar Xilosa-lisina-tergitol (XLT4, DB). Las placas de VBS y XLT4 se incubaron a $35{ }^{\circ} \mathrm{C} \pm 2{ }^{\circ} \mathrm{C}$ durante 24 a $48 \mathrm{~h}$. De cada agar se seleccionaron tres colonias con morfología típica de Salmonella y se sometieron a pruebas bioquímicas en agar triple azúcar y hierro y en agar hierro y lisina (BD) a $35 \pm 2{ }^{\circ} \mathrm{C}$ por $24 \mathrm{~h}$. Los aislamientos que mostraron el perfil bioquímico típico de Salmonella se confirmaron por la amplificación de invA y fimA por $\mathrm{PCR}^{(13)}$. Cincuenta (50) cepas con morfología y perfil bioquímico típico de Salmonella y confirmadas por PCR, se seleccionaron al azar considerando el envío de una cepa presuntamente positiva por muestra, al Instituto de Diagnóstico y Referencia Epidemiológicos (InDRE) para su confirmación y serotipificación por el método descrito por Kauffmann ${ }^{(14)}$. La comparación entre el número de aislamientos de Salmonella confirmados por el InDRE en cada rastro por mes de aislamiento, se analizó mediante la prueba de análisis de varianza (ANOVA), con el programa estadístico GraphPad Prism $8^{(15)}$.

El perfil de susceptibilidad a 10 agentes antimicrobianos de las cepas aisladas de Salmonella, se determinó siguiendo la técnica de Kirby-Bauer, estandarizada por el Clinical and Laboratory Standards Institute ${ }^{(16)}$. Los antimicrobianos evaluados fueron ampicilina ( $\mathrm{AM}, 10 \mu \mathrm{g}$ ), ácido nalidíxico (NA, $30 \mu \mathrm{g}$ ), cefalotina (CF, $30 \mu \mathrm{g}$ ), ceftriaxona (CRO, $30 \mu \mathrm{g}$ ), ciprofloxacino (CIP, $30 \mu \mathrm{g}$ ), cloranfenicol (C, $30 \mu \mathrm{g}$ ), estreptomicina (S, $10 \mu \mathrm{g}$ ), gentamicina (GM, $10 \mu \mathrm{g}$ ), kanamicina $(\mathrm{K}, 30 \mu \mathrm{g})$, tetraciclina (TE, $30 \mu \mathrm{g}) \mathrm{y}$ trimetoprima-sulfametoxazol (SXT, 1.25 y $23.75 \mu \mathrm{g}$ ). La medición de los halos de inhibición (mm) se interpretaron de acuerdo a las tablas del CLSI ${ }^{(17)}$. Escherichia coli ATCC 25922 fue utilizada como control.

Se analizó por PCR la presencia de los genes de resistencia tetA y tet $B^{(18)}$ en todos los aislamientos que mostraron resistencia a TE, y de las integrasas 1 (intI1) y 2 (intI2), en las cepas que resultaron multirresistentes a tres o mas clases de antimicrobianos ${ }^{(17)}$ siguiendo un protocolo previamente establecido ${ }^{(18)}$. 


\section{Resultados y discusión}

Los aislamientos de Salmonella observados en el rastro TIF y en el rastro RNC fueron de $1.3 \%$ (1/80) y $46.8 \%$ (37/79), respectivamente. El análisis de ANOVA realizado muestra diferencias significativas $(P<0.05)$ entre el número de aislamientos obtenidos entre los meses de octubre, enero y febrero, no siendo así para los meses de marzo y abril (Cuadro 1). Estos resultados sugieren que las medidas de control implementadas en el rastro TIF disminuyen significativamente el número de aislamientos de Salmonella en las canales que ahí se procesan, así como el hecho de que sólo se procesan cerdos en dicho rastro. De acuerdo con la EFSA, la implementación de medidas de control en los procesos de sacrificio del ganado porcino tiene un gran impacto en la reducción de la presencia bacterias patógenas que causan gastroenteritis en las canales ${ }^{(4)}$. La cantidad de aislamientos de Salmonella observada en las canales del rastro RNC puede estar relacionada con fallas en el proceso de evisceración. Existe evidencia que demuestra que después de la evisceración del ganado porcino, la contaminación por enterobacterias aumenta en la canal y la cantidad de éstas es diferencial en cada una de las partes de la canal $^{(5)}$, aumentando la contaminación bacteriana en la áreas anteriores y ventrales de la canal cuando hay cortes en las amígdalas durante la remoción de las vísceras, y disminuye cuando las herramientas utilizadas en la remoción de éstas son higienizados posterior a la remoción de las amígdalas ${ }^{(5)}$. Así mismo, la contaminación observada en el rastro RNC puede ser originada por la contaminación cruzada entre las bacterias presentes en el ganado porcino y bovino, debido a que en el rastro RNC evaluado en este estudio, también se lleva a cabo el sacrificio de ganado bovino ${ }^{(19)}$.

Cuadro 1: Serotipo, mes de aislamiento y perfil de resistencia de 38 cepas Salmonella enterica aisladas de canales de cerdo en dos tipos de rastros

\begin{tabular}{cccc}
\hline Tipo de rastro & Serotipo & $\begin{array}{c}\text { Mes de } \\
\text { aislamiento }\end{array}$ & Perfil de resistencia \\
\hline TIF & Typhimurium & Abril & GM, K, S, C, NA, TE \\
\hline \multirow{2}{*}{ Agona } & Febrero & K, S \\
& Febrero & K \\
& Febrero & K \\
& Enero & K, NA, STX, TE \\
& Octubre & K, SXT, TE \\
& Enero & K, NA, TE \\
& Enero & K, NA \\
& Enero & K, S \\
& Enero & K \\
& Bovismorbificans & Octubre & K, S, C, TE \\
\hline Bredeney & Octubre & K \\
Derby & Octubre & K \\
& Enero & K, S, AM, C, TE \\
& & Febrero & K, S, NA, TE \\
& & Febrero & KA, TE
\end{tabular}




\begin{tabular}{|c|c|c|c|}
\hline \multirow{21}{*}{ RNC } & \multirow{14}{*}{ London } & Octubre & $\mathrm{K}, \mathrm{NA}, \mathrm{TE}$ \\
\hline & & Octubre & $\mathrm{K}, \mathrm{NA}, \mathrm{TE}$ \\
\hline & & Enero & $\mathrm{K}, \mathrm{NA}, \mathrm{TE}$ \\
\hline & & Enero & $\mathrm{K}, \mathrm{NA}, \mathrm{TE}$ \\
\hline & & Enero & $\mathrm{K}, \mathrm{NA}, \mathrm{TE}$ \\
\hline & & Febrero & $\mathrm{K}, \mathrm{NA}, \mathrm{TE}$ \\
\hline & & Febrero & $\mathrm{K}, \mathrm{NA}, \mathrm{TE}$ \\
\hline & & Marzo & $\mathrm{K}, \mathrm{NA}, \mathrm{TE}$ \\
\hline & & Octubre & $\mathrm{K}, \mathrm{TE}$ \\
\hline & & Enero & $\mathrm{K}, \mathrm{TE}$ \\
\hline & & Enero & $\mathrm{K}, \mathrm{TE}$ \\
\hline & & Enero & $\mathrm{K}, \mathrm{TE}$ \\
\hline & & Febrero & $\mathrm{K}, \mathrm{TE}$ \\
\hline & & Febrero & $\mathrm{K}, \mathrm{TE}$ \\
\hline & Montevideo & Octubre & $\mathrm{K}$ \\
\hline & \multirow{3}{*}{ Muenchen } & Febrero & $\mathrm{K}, \mathrm{C}, \mathrm{NA}, \mathrm{TE}$ \\
\hline & & Enero & $\mathrm{K}, \mathrm{S}, \mathrm{C}, \mathrm{TE}$ \\
\hline & & Enero & $\mathrm{K}, \mathrm{NA}, \mathrm{TE}$ \\
\hline & \multirow[t]{2}{*}{ Typhimurium } & Octubre & $\mathrm{GM}, \mathrm{K}, \mathrm{S}, \mathrm{C}, \mathrm{NA}, \mathrm{TE}$ \\
\hline & & Octubre & $\mathrm{K}, \mathrm{S}, \mathrm{C}, \mathrm{SXT}, \mathrm{TE}$ \\
\hline & Senftenberg & Octubre & $\mathrm{K}, \mathrm{C}, \mathrm{TE}$ \\
\hline
\end{tabular}

TIF= Tipo Inspección federal; $\mathrm{RNC}=$ rastro no certificado de competencia municipal, $\mathrm{GM}=$ gentamicina, $\mathrm{K}=$ kanamicina,, $\mathrm{S}=$ estreptomicina, $\mathrm{C}=$ cloranfenicol, $\mathrm{NA}=$ ácido nalidixico $\mathrm{TE}=$ tetraciclina, $\mathrm{SXT}=$ trimetoprima-sulfametoxazol $\mathrm{AM}=$ ampicilina.

Los serotipos aislados en el rastro RNC fueron Salmonella London (45.9 \%, 17/37), Anatum (16.2 \%, 6/37), Agona y Muenchen (8.1 \% cada una, 3/37), Derby y Typhimurium (5.4\%, 2/37), Bredeney, Bovismorficans, Montevideo y Senftenberg (2.7 \% cada una, 1/37), mientras que el único serotipo aislado en el rastro TIF fue $S$. Typhimurium (Cuadro 1). Los serotipos predominantes en reportes previos de aislamientos son diversos y dependen del año y el lugar, así como del tipo de alimento analizado. En muestras de canales de cerdo de Estados Unidos, colectadas de 2003 al 2015, los cinco serotipos predominantes fueron $S$. Typhimurium monofásica (4,(5),12:i:-), Infantis, Johannesburg, Typhimurium y Derby ${ }^{(20)}$. En Bélgica, en un estudio realizado entre octubre del 2015 y febrero del 2016, S. Typhimurium, Derby, Livingstone, Rissen y Bredeney fueron los serotipos prevalentes ${ }^{(5)}$, mientras que en España, en un estudio realizado entre noviembre de 2012 y mayo de 2014, los serotipos aislados fueron $S$. Rissen, Typhimurium, Panama y Brandenburg ${ }^{(6)}$. En contraste, en la República de China, en el análisis realizado de octubre de 2012 a julio de 2013, los serotipos encontrados fueron $S$. Saintpaul, Agona, Give y Corvallis, y en menor proporción Derby e Infantis ${ }^{(21)}$. Los serotipos $S$. Agona, Typhimurium y Derby encontrados en el presente estudio coinciden con aquellos recuperados de canales de cerdo en muestreos realizados en los países mencionados.

En la reunión organizada en conjunto por la FAO y la OMS en junio del 2018, expertos en el área discutieron al respecto de la resistencia antimicrobiana en patógenos aislados de alimentos y establecieron como un área prioritaria, la investigación de bacterias 
resistentes a antimicrobianos y genes de resistencia, con énfasis en la búsqueda de patógenos resistentes a antimicrobianos que son responsables de causar enfermedades por el consumo de alimentos ${ }^{(2)}$.

Debido a que el estudio de la resistencia de Salmonella a los antimicrobianos es de interés mundial, se analizó la susceptibilidad de los 38 aislamientos obtenidos ( 37 del rastro RNC y 1 del rastro TIF) a 10 agentes antimicrobianos. Los resultados muestran que los 38 aislamientos fueron resistentes a $\mathrm{K}$ y susceptibles a CIP y a las cefalosporinas evaluadas (CF y CRO), resaltando que el $100 \%$ de los aislamientos fueron resistente al menos a una clase de antimicrobianos. El $73.7 \%(28 / 38)$ de los aislamientos fueron resistentes a TE, el $44.7 \%$ a NA (17/38), $23.7 \%$ a S (9/38), $21.1 \%$ a C (8/38), $7.9 \%$ a SXT (3/38), $5.3 \%$ a GM (2/38) y $2.6 \%$ a AM (1/38). Los aislamientos de $S$. Derby, Bovismorficans y Montevideo sólo mostraron resistencia a K (Figura 1).

Figura 1: Porcentaje de cepas de Salmonella enterica aisladas de canales de cerdo que resultaron resistentes a diversas clases de antimicrobianos evaluados

\section{Clases de \\ antimicrobianos}

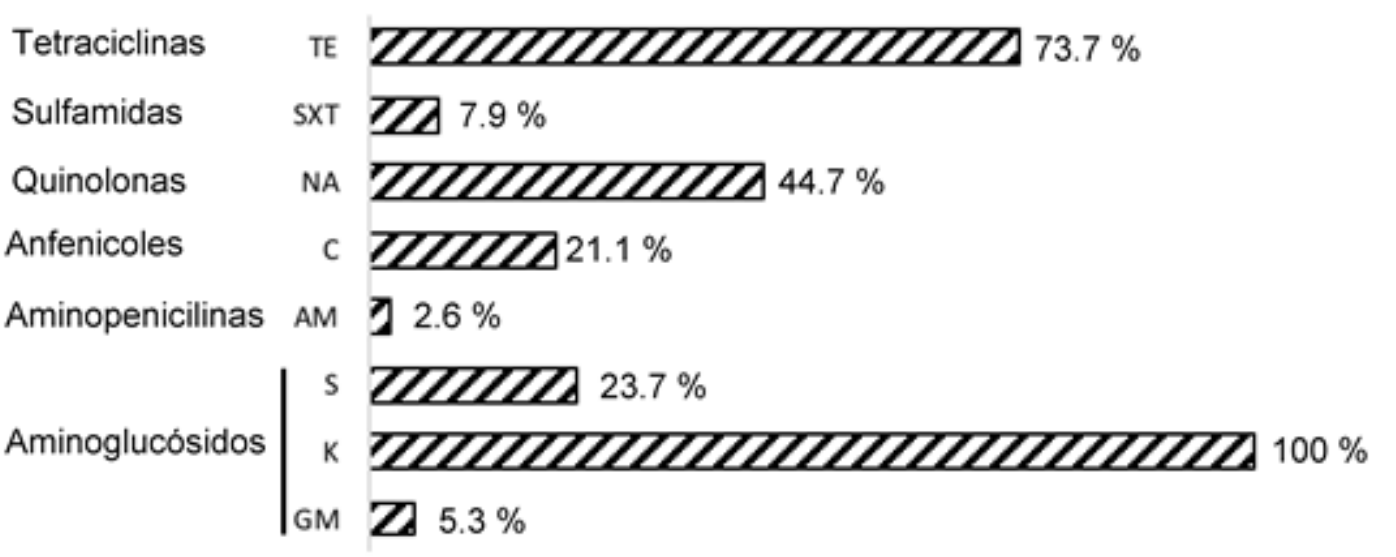

Algunos de los aislamientos de $S$. Agona (3), Anatum (4), Bovismorbificans (1), Derby (2), London (6) y Montevideo (1) presentaron resistencia a uno o dos clases de antimicrobianos. En contraste, los otros aislamientos de $S$. Anatum (3), Bredeney (1), London (11), Muenchen (3), Typhimurium (3) y Senftenberg (1) mostraron resistencia a tres y/o cuatro clases de antimicrobianos (Cuadro 2), siendo los aminoglucósidos, las tetraciclinas y la ciprofloxacina, las clases y el antimicrobiano a los cuales se observó mayor resistencia (Figura 1). En Estados Unidos, el NARMS reportó un incremento en la resistencia contra fluoroquinolonas (CIP), cefalosporinas de tercera generación (CF) y macrólidos (azitromicina), antimicrobianos que se usan de manera frecuente en el tratamiento de las infecciones producidas por S. Typhi, Paratyphi y Salmonella no tifoidea $^{(3)}$. En contraste, en los aislamientos obtenidos de rastros de sacrificio de cerdos y de carne vendida en expendios en China, se observó resistencia a las tetraciclinas, aminopenicilinas (AM), sulfamidas (SXT) y aminoglucósidos $(\mathrm{S})^{(21)}$. 
Cuadro 2: Número de aislamientos resistentes de serotipos de Salmonella enterica aislados de canales de cerdos en dos tipos de rastros

\begin{tabular}{lllllll}
\hline & & \multicolumn{7}{c}{ No. de clases de antimicrobianos } \\
Serotipo & No. & $\mathbf{\%}$ & $\mathbf{1}$ & $\mathbf{2}$ & $\mathbf{3}$ & $\mathbf{4}$ \\
\hline Agona & 3 & 7.9 & 3 & & & \\
Anatum & 6 & 15.8 & 2 & 1 & 2 & 1 \\
Bovismorbificans & 1 & 2.6 & 1 & & & \\
Bredeney & 1 & 2.6 & & & 1 & \\
Derby & 2 & 5.3 & & 2 & & \\
London & 17 & 44.7 & & 6 & 10 & 1 \\
Montevideo & 1 & 2.6 & 1 & & & \\
Muenchen & 3 & 7.9 & & & 2 & 1 \\
Typhimurium & 3 & 7.9 & & & & 3 \\
Senftenberg & 1 & 2.6 & & & 1 & \\
\hline TOTAL & 38 & 100 & 7 & 9 & 16 & 6 \\
\hline
\end{tabular}

La resistencia observada a los diferentes agentes antimicrobianos es difícil de comparar entre diferentes estudios, debido a que ésta se ve influenciada por el tipo agentes utilizados en la región durante en crecimiento del ganado porcino ${ }^{(6)}$, así como por la presencia de los residuos de antimicrobianos en el agua, el suelo de las granjas o rastros donde se desarrollan o sacrifican los animales ${ }^{(2)}$.

El $73.7 \%$ (28/38) de los aislamientos fueron resistentes a tetraciclina, por ello se determinó la presencia de tetA y tetB por PCR, dos de los genes que confieren la resistencia a $\mathrm{TE}^{(6)}$. Sin que se observara amplificación de estos genes en ninguno de los aislamientos resistentes a TE. Otro agente al que un gran número de aislamientos mostró resistencia fue NA (44.7\%). La resistencia a este antimicorbiano es relevante porque disminuye la susceptibilidad a CIP, agente que es usado comúnmente en el tratamiento de la salmonelosis ${ }^{(3)}$. La resistencia a AM-C-S-SXT-TE y AM-S-SXT-TE (fenotipos ACSSuT y ASSuT, respectivamente) es buscada comúnmente en Salmonella no tifoideas, debido a las complicaciones encontradas en el tratamiento de la salmonelosis ${ }^{(3)}$. En este estudio no se encontraron aislamientos de Salmonella con esos fenotipos específicos, pero sí se encontraron cepas con resistencia a cuatro clases de antimicrobianos, lo cual también puede representar un problema en el tratamiento de la enfermedad producida en humanos (Cuadros 1 y 2).

La adquisición de elementos de resistencia es a través de la transferencia horizontal de genes o grupos de genes y es mediada por elementos genéticos móviles (integrones, plásmidos $\mathrm{o}$ transposones $)^{(6,22)}$. Los integrones son sistemas de recombinación constituídos principalmente por una integrasa, un sitio de recombinación y un promotor fuerte. En enterobacterias, tres clases de integrones están asociados a la adquisión de casetes de resistencia; la clase 1 se asocia con resistencia a la mayoría de los $\beta$-lactámicos conocidos, aminoglicósidos, trimetropima, rifampicina, cloranfenicol, quinolonas, 
eritromicina y compuestos cuternarios de amonio $^{(22)}$. Seis de los aislamientos que mostraron resistencia a 3 ó 4 clases de antimicrobianos, se seleccionaron para la detección de integrones clase 1 y 2, encontrando que cuatro aislamientos de los serotipos Anatum (1), London (1) y Typhimurium (2) mostraron la amplificación del gen intI1 (integron clase 1). Se ha reportado que el integron clase 1 es el más frecuente en aislamientos resistentes de patógenos como Klebsiella, Salmonella, Shigella y Yersinia ${ }^{(22)}$. Ninguna banda de amplificación para los genes intI1 y intI2 se observó en las cepas aisladas de los serotipos Bredeny y Muenchen (Cuadro 3) por lo que el perfil de resistencia observado puede deberse a un integron clase 3 , o a otro elemento móvil.

Cuadro 3: Amplificación de los genes intl1 e intl2 en aislamientos de Salmonella enterica obtenidos de canales de cerdo en dos tipos de rastros

\begin{tabular}{lllll}
\hline Origen & Serotipo & $\begin{array}{l}\text { Perfil de resistencia a } \\
\text { antimicrobianos }\end{array}$ & $\begin{array}{l}\text { Detección } \\
\text { de intII }\end{array}$ & $\begin{array}{l}\text { Detección } \\
\text { de intI2 }\end{array}$ \\
\hline Rastro TIF & Typhimurium & GM, K, S, C, NA, TE & + & - \\
\hline Rastro RNC & Anatum & K, NA, SXT, TE & + & - \\
& Bredeney & K, S, C, TE & - & - \\
& London & K, S, AM, C, TE & + & - \\
& Muenchen & K, C, NA, TE & - & - \\
& Typhimurium & $\mathrm{K}, \mathrm{S}, \mathrm{C}, \mathrm{SXT}, \mathrm{TE}$ & + & - \\
\hline
\end{tabular}

$\mathrm{TIF}=$ Tipo Inspección federal; $\mathrm{RNC}=$ rastro no certificado de competencia municipal.

$\mathrm{AM}=$ ampicilina, $\mathrm{C}=$ cloranfenciol, $\mathrm{GM}=$ gentamicina, $\mathrm{K}=$ kanamicina, $\mathrm{NA}=$ ácido nalidixico, $\mathrm{S}=$ estreptomicina, TE= tetraciclina, $\mathrm{SXT}=$ trimetropima con sulfametoxasol.

\section{Conclusiones e implicaciones}

Este estudio evidencia la presencia de cepas de Salmonella resistentes a diferentes clases de antimicrobianos en canales de cerdo. La presencia de estas cepas multirresistentes en la carne de cerdo destinada para consumo de la población, podría causar un problema de salud pública, debido a que, al ser consumida en alimentos con prácticas deficientes en el manejo, puede ocasionar una infección de difícil tratamiento. Así mismo, enfatiza la importancia de implementar buenas prácticas de manufactura y medidas de control durante el proceso de sacrificio y obtención de la carne para reducir la presencia de Salmonella y como consecuencia la diseminación de elementos móviles portadores de genes de resistencia hacia otro tipo de enterobacterias. No obstante, los hallazgos encontrados, este estudio presenta limitaciones con respecto a la evaluación y verificación del proceso de higienización llevado a cabo en cada rastro, por lo que la conclusión relacionada a que, la implementación de buenas prácticas de manufactura reduce la presencia de Salmonella, se realizó con base a la información obtenida en otros estudios $^{(23)}$. Así mismo, los resultados mostrados son inherentes a los rastros estudiados y no hay datos suficientes para inferir en qué grado estos representan la situación de los rastros RNC y TIF en la zona o el país. 


\section{Agradecimientos}

Agradecemos profundamente el trabajo realizado en este proyecto por el M. en C. Carlos Pacheco Gallardo, quien falleció el 14 de octubre del 2019. Apreciamos la asistencia técnica en la recolección y procesamiento de muestras a Diana Arcelia Castro de Sales, María Guadalupe Pilar Soto, José Antonio Vilchis Carmona y Manuel Alejandro Cortez Gómez.

\section{Literatura citada:}

1. Karkey A, Thwaites GE, Baker S. The evolution of antimicrobial resistance in Salmonella Typhi. Curr Opin Gastroenterol 2018;34(1):25-30.

2. FAO/WHO. Food and Agriculture Organization of the United Nations/ World Health Organization. FAO/WHO expert meeting on foodborne antimicrobial resistance: Role of environment, crops and biocides. Rome, Italy. 2018.

3. CDC. National Antimicrobial Resistance Monitoring System for Enteric Bacteria (NARMS). Human Isolates Surveillance Report for 2015. Atlanta, Georgia: U.S. Department of Health and Human Services, Centers for Disease Control, CDC. 2018.

4. EFSA. European Food Safety Authority. Scientific opinion on a quantitative microbiological risk assessment of Salmonella in slaughter and breeder pigs. Parma, Italy. 2010.

5. Biasino W, De Zutter L, Mattheus W, Bertrand S, Uyttendaele M, Van Damme I. Correlation between slaughter practices and the distribution of Salmonella and hygiene indicator bacteria on pig carcasses during slaughter. Food Microbiol 2018;70:192-199.

6. Cameron-Veas K, Fraile L, Napp S, Garrido V, Grillo MJ, Migura-Garcia L. Multidrug resistant Salmonella enterica isolated from conventional pig farms using antimicrobial agents in preventative medicine programs. Vet J 2018;234:36-42.

7. Consejo Mexicano de la Carne. Compendio Estadístico 2017. http://comecarne.org/wp-content/uploads/2018/05/CompendioEstad\%C3\%ADstico-2017-v7-1-sin-elab.pdf. Consultado 20 Ene, 2019.

8. CIMA. Centro de Información de Mercados Agroalimentarios \& ASERCA. Agencia de Servicios a la Comercialización y Desarrollo de Mercados Agropecuarios. Reporte del Mercado de Carne de Porcino. 2018. https://www.cima.aserca.gob.mx/work/models/cima/pdf/cadena/2018/Reporte_mer cado_porcino_290618.pdf. Consultado 20 Ene, 2020. 
9. SADER. Secretaría de Agricultura y Desarrollo Rural. Rastros no certificados. https://www.gob.mx/senasica/acciones-y-programas/rastros-no-certificados. Consultado: 20 Ene, 2020.

10. SADER. Secretaría de Agricultura y Desarrollo Rural. Rastros Tipo Inspección Federal. https://www.gob.mx/firco/articulos/sabes-que-es-un-rastro-tipoinspeccion-federal?idiom=es. Consultado: 20 Ene, 2020.

11. S.S. Secretaría de Salud. Norma Oficial Mexicana NOM-194-SSA1-2004, Productos y servicios. Especificaciones sanitarias en los establecimientos dedicados al sacrificio y faenado de animales para abasto, almacenamiento, transporte y expendio. Especificaciones sanitarias de productos. Estados Unidos Mexicanos: S.S. 2004.

12. USDA. United State Department of Agriculture. Food Safety and Inspection Service. Isolation and identification of Salmonella from meat, poultry and eggs products, MLG 4.04. Isolation and identification of Salmonella from meat, poultry and eggs products, MLG 4.04. Athens, GA. USA: Food Safety and Inspection Service. Laboratory QA/QC Division; 2008.

13. Perez-Montano JA, Gonzalez-Aguilar D, Barba J, Pacheco-Gallardo C, CamposBravo CA, Garcia S, et al. Frequency and antimicrobial resistance of Salmonella serotypes on beef carcasses at small abattoirs in Jalisco State, Mexico. J Food Prot 2012;75(5):867-73.

14. Kauffmann F. On the history of Salmonella research. Zentralblatt fur Bakteriologie, Parasitenkunde, Infektionskrankheiten und Hygiene 1 Abt Medizinisch-hygienische Bakteriologie, Virusforschung und Parasitologie Originale 1966;201(1):44-8.

15. GraphPad. Computer software GraphPad Prism8. Computer software GraphPad Prism8. San Diego, CA: Digital Millennium; 2018.

16. CLSI. Clinical and Laboratory Standards Institute. Performance standards for antimicrobial disk susceptibility test. M2-A9. Wayne, PA, USA: CLSI; 2006.

17. CLSI. Clinical and Laboratory Standards Institute. Performance standards for antimicrobial susceptibility testing; 27 $7^{\text {th }}$ Informational Suppl. M100-S27. CLSI, Wayne, PA, USA; 2017.

18. Lapierre L, Cornejo J, Borie C, Toro C, San Martin B. Genetic characterization of antibiotic resistance genes linked to class 1 and class 2 integrons in commensal strains of Escherichia coli isolated from poultry and swine. Microb Drug Resist 2008;14(4):265-72.

19. Bersisa A, Tulu D, Negera C. Investigation of bacteriological quality of meat from abattoir and butcher shops in Bishoftu, Central Ethiopia. Int J Microbiol 2019;641683. 
20. Yuan C, Krull A, Wang C, Erdman M, Fedorka-Cray PJ, Logue CM, et al. Changes in the prevalence of Salmonella serovars associated swine production and correlations of avian, bovine and swine-associated serovars with human-associated serovars in the United States (1997-2015). Zoonoses Public Health. 2018.

21. Li Y, Cai Y, Tao J, Kang X, Jiao Y, Guo R, et al. Salmonella isolated from the slaughterhouses and correlation with pork contamination in free market. Food Control 2016;59:591-600.

22. Kaushik M, Kumar S, Kapoor RK, Virdi JS, Gulati P. Integrons in Enterobacteriaceae: diversity, distribution and epidemiology. Int J Antimicrob Agents 2018;51(2):167176.

23. Fajardo-Guerrero M, Rojas-Quintero C, Chamorro-Tobar I, Zambrano C, Sampedro F, Carrascal-Camacho AK. Exposure assessment of Salmonella spp. in fresh pork meat from two abattoirs in Colombia. Food Sci Technol Int 2019:1082013219864746. 